\title{
$\alpha$-relaxation in a supercooled binary mixture
}

\author{
M. Fuchs and A. Latz ${ }^{1}$ \\ Physik-Department, Technische Universität München, W-85748 Garching, Germany
}

\begin{abstract}
The $\alpha$-master functions of the mode coupling theory for a binary soft-sphere mixture are presented. They are directly determined by the static structure factors $S_{q}$. The asymmetric stretching of the susceptibility spectra, which is mainly caused by the common von Schweidler high frequency asymptote, is well described by Kohlrausch fits. The stretching parameters and $\alpha$-relaxation times for various correlation functions are discussed as functions of the wave vector. For small wave vectors two peaks appear in the density-density spectrum due to an interference of the inter-diffusion pole and the Mountain peak.
\end{abstract}

\section{Introduction}

Supercooled liquids exhibit slow, non-exponential relaxation processes labelled with Greek letters. The $\alpha$-process describes the final decay into equilibrium.

The mode coupling theory (MCT) of the glass transition has opened the possibility to calculate $\alpha$-relaxation curves starting from the equilibrium structure of the liquid; for reviews see $[1,2]$ and references cited therein. A critical temperature $T_{\mathrm{c}}$ has been predicted separating liquid and glass and asymptotic dynamical scaling laws have been derived close to $T_{c}$. Neglecting thermally activated transport processes $[3,4]$ a one parameter scaling law for the $\alpha$-process has been derived for temperatures close to and above $T_{\mathrm{c}}$ [1],

$$
\Phi_{X}(t)=\hat{\Phi}_{X}(\hat{t}), \quad T \rightarrow T_{\mathrm{c}}+, \quad \tau \rightarrow \infty, \quad \hat{t}=t / \tau=\text { const } .
$$

Within the $\alpha$-relaxation regime an autocorrelation function $\Phi_{X}(t)$ of a variable $X$ is given by a temperature independent master function varying with rescaled time $\hat{t}=t / \tau(T)$ on the divergent time scale $\tau, \tau \rightarrow \infty$ for $T \searrow T_{\mathrm{c}}$. The functional form of the $\alpha$-master curves $\hat{\Phi}_{X}(\hat{t})$ depends on the equilibrium structure of the liquid. The approximations of the MCT can most sensitively be tested with this dynamical process.

\footnotetext{
${ }^{1}$ Present address: Institute for Physical Science, University of Maryland, College Park, MD 20742, USA.
} 
For short rescaled times $\hat{t}$ the common von Schweidler power law [1],

$$
\hat{\Phi}_{X}(\hat{t})=f_{X}^{\mathrm{c}}-h_{X} \hat{t}^{b}+\mathcal{O}\left(\hat{t}^{2 b}\right), \quad 0<b \leqslant 1,
$$

shows that the $\alpha$-master functions are non-exponentional in general. Already with schematic models MCT calculations have shown that the Kohlrausch functions,

$$
\phi_{X}^{\mathrm{K}}(\hat{t})=f_{X}^{\mathrm{K}} \exp \left[-\left(\hat{t} / \hat{\tau}_{X}\right)^{\beta_{X}}\right],
$$

provide rather good fits to $\alpha$-master curves with stretching exponents $\beta_{X}$ depending on the variable $X$ and showing systematic deviations due to $\beta_{X} \neq b$ [5].

The $\alpha$-master curves for density fluctuations in a hard sphere system (HSS) have been calculated using the static structure factor $S_{q}$ as only input [6]. The $\alpha$-relaxation strengths, the relaxation times and further characteristics of the functional shapes have successfully been tested as functions of wave vector $q$ and (packing-) density $\varphi$ using dynamic light scattering measurements in colloidal suspensions [7].

The glass transition of a soft sphere binary mixture has extensively been studied by molecular dynamics simulations (MD); see [8-11] and references cited therein. The calculated and simulated glass transition points lie rather close [12]. The $\alpha$-relaxation strength as predicted by the MCT [12] compares favorably to the MD results [10]. The predicted $\beta$-relaxation curves of the MCT without thermally activated processes can describe the dynamics on an intermediate time window of about 2 decades [13]. In the present paper the $\alpha$-master functions for the binary system are presented. This makes possible further quantitative tests of the MCT using MD data.

The studied binary mixture is composed of spheres of diameter $d_{i}, i=1,2$, interacting via the potential

$$
V_{i j}=\varepsilon\left(d_{i j} / r\right)^{12}, \quad d_{i j}=\frac{1}{2}\left(d_{i}+d_{j}\right) .
$$

As in the MD simulations $d_{2} / d_{1}=1.2$ and an equimolar mixture, $x_{1}=x_{2}=\frac{1}{2}$, are chosen [9-11]. The thermodynamic state of the mixture is then specified by the single parameter $\Gamma[8]$,

$$
\Gamma=n\left(x_{1}^{2} d_{1}^{3}+2 x_{1} x_{2} d_{12}^{3}+x_{2}^{2} d_{2}^{3}\right)\left(\varepsilon / k_{\mathrm{B}} T\right)^{1 / 4} .
$$

The dynamical information is contained in the partial density fluctuation functions $F_{q}^{i j}(t)=(1 / N)\left\langle\varrho_{q}^{i}(t)^{*} \varrho_{q}^{i}\right\rangle, i, j=1,2$, which reduce to the partial structure factors $S_{q}^{i j}$ at time $t=0$ [14]. The structure factors $S_{q}^{i j}$ of this system 
can be obtained from the Rogers-Young scheme [15]. A convenient length scale $a$ is given by the total density $n$ via $\frac{4}{3} \pi n a^{3}=1$.

Section 2 collects the equations for the $\alpha$-relaxation of a binary mixture with short range interactions. In section 3 the results are presented and in section 4 compared to the MD data.

\section{Basic equations}

The MCT equations for binary mixtures deal with the $2 \times 2$ matrix $F_{q}(t)$ of partial density fluctuations $F_{q}^{i j}(t)[16,17]$. Thermal fluctuations are assumed to be decoupled. The $\alpha$-scaling equations for the $\alpha$-master functions $\hat{F}_{q}(\hat{t})$ neglecting thermally activated processes can be derived in the same way as in the one component case [1],

$$
q^{2} \hat{F}_{q}(\hat{t})=S_{q}\left(\hat{M}_{q}(\hat{t}) S_{q}-\frac{\mathrm{d}}{\mathrm{d} \hat{t}} \int_{0}^{\hat{t}} \mathrm{~d} t^{\prime} \hat{M}_{q}\left(\hat{t}-t^{\prime}\right) \hat{F}_{q}\left(t^{\prime}\right)\right) .
$$

The carets will be dropped in the following as only $\alpha$-quantities will be discussed. Eq. (6a) describes the dynamics on the diverging time scale $\tau$, $\tau \propto \sigma^{-\gamma}$ for $\sigma \rightarrow 0$ as $\Gamma \searrow \Gamma^{\mathrm{c}}$. It is closed by the following expression for the memory functions $[18,12]$ :

$$
M_{q}^{i j}(t)=\frac{1}{x_{i} x_{j}} \int \frac{\mathrm{d}^{3} k}{(2 \pi)^{3}} \sum_{\alpha \beta} \sum_{\alpha^{\prime} \beta^{\prime}} V^{i \alpha \beta}(q, k) V^{i \alpha^{\prime} \beta^{\prime}}(q, q-k) F_{k}^{\alpha \alpha^{\prime}}(t) F_{|q-k|}^{\beta \beta^{\prime}}(t),
$$

with the vertices determined by the direct correlation function $c_{q}^{i j}=\delta_{i j} / x_{i}-$ $\left(S_{q}^{-1}\right)_{i j}[12]$,

$$
V^{i \alpha \beta}(q, k)=\frac{q \cdot k}{q} c_{k}^{i \alpha} \delta^{i \beta}+\frac{q \cdot(q-k)}{q} c_{|q-k|}^{i \beta} \delta^{i \alpha} .
$$

The equilibrium structure $S_{q}^{i j}$ in eq. (6) is evaluated at the critical point $\Gamma^{\mathrm{c}}$. The exponent parameter $\lambda$ is also determined by $S_{q}^{i j}\left(\Gamma^{c}\right)$ [12] and from it the von Schweidler exponent $b$ follows, which appears in the power law short time expansion of the $\alpha$-master functions [1],

$$
F_{q}^{i j}(t)=f_{q}^{i j}-h_{q}^{i j} t^{h}+h_{q}^{(2) i j} t^{2 h}-h_{q}^{(3) i j} t^{3 h}+\cdots .
$$


As for a simple liquid a (normal) critical point $\Gamma^{\mathrm{c}}$ is defined by the occurrence of a codimension 1 fold singularity in the equations for the $\alpha$-relaxation strength $f_{q}$ [1]. More complicated transition scenarios (codimension 2 and larger), which may occur due to negative non-diagonal vertices in (6b), are not considered in this paper.

The $\alpha$-equations for the self correlation functions $F_{q}^{(s) i}(t)$ for a particle of species $i$ differ only little from the one component case [18],

$$
\begin{aligned}
& q^{2} F_{q}^{(\mathrm{s}) i}(t)=M_{q}^{(\mathrm{s}) i}(t)-\frac{\mathrm{d}}{\mathrm{d} t} \int_{0}^{t} \mathrm{~d} t^{\prime} M_{q}^{(\mathrm{s}) i}\left(t-t^{\prime}\right) F_{q}^{(\mathrm{s}) i}\left(t^{\prime}\right), \\
& M_{q}^{(\mathrm{s}) i}(t)=\int \frac{\mathrm{d}^{3} k}{(2 \pi)^{3}} n\left(\frac{q \cdot \boldsymbol{k}}{q}\right)^{2}(c F)_{k}^{(i)}(t) F_{q-k}^{(\mathrm{s}) i}(t), \\
& (c F)_{q}^{(i)}(t)=\left(c_{q}^{i i}\right)^{2} F_{q}^{i i}(t)+2 c_{q}^{i i} c_{q}^{i j} F_{q}^{i j}(t)+\left(c_{q}^{i j}\right)^{2} F_{q}^{j j}(t), \quad j \neq i .
\end{aligned}
$$

The procedure for solving (6) and (8) is analogous to the one used and described in [5] and [6]. The discretization of the wave vector integrals in eq. (6b), $q a=0,0.1, \ldots, 29.9$, which was also used and discussed in [6], leads to a system of 900 coupled equations.

A discussion of the dynamics of mixtures is simplified by looking at fluctuations of the total density $\delta Q_{q}^{\mathrm{n}}=\delta \mathrm{Q}_{q}^{1}+\delta \mathrm{Q}_{q}^{2}$ and of the concentration, which for $x_{1}=x_{2}$ can be defined by $\delta Q_{q}^{c}=\delta Q_{q}^{1}-\delta Q_{q}^{2}$; in the following advantage of $x_{1}=x_{2}=\frac{1}{2}$ is taken, the generalization is straightforward. The $\alpha$ equations for the $F^{r}, r=\mathrm{nn}, \mathrm{nc}$, cc, take on the identical form as (6a), if the correspondingly transformed memory functions $\boldsymbol{M}^{r}$ for the fluctuating forces, $\delta f_{q}^{\mathrm{n}}=\frac{1}{2}\left(\delta f_{q}^{1}+\delta f_{q}^{2}\right)$ and $\delta f_{q}^{\mathrm{c}}=\frac{1}{2}\left(\delta f_{q}^{1}-\delta f_{q}^{2}\right)$, are used. For small wave vectors hydrodynamic conservation laws result in the following simplification of the memory functions [14]:

$$
M_{q}^{i j}(t) \rightarrow(-1)^{i+j} M_{0}^{\text {cc }}(t)+\mathcal{O}\left(q^{2}\right), \quad \text { for } q \rightarrow 0 .
$$

The factorization approximation of the MCT obeys this condition with the result for $M_{0}^{\text {cc }}$,

$$
M_{0}^{\mathrm{cc}}(t)=\frac{2}{3 \pi^{2} n} \int_{0}^{\infty} \mathrm{d} k k^{4}\left(c_{k}^{12}\right)^{2} \operatorname{Det}\left[F_{k}(t)\right] .
$$

A generalized inter-diffusion coefficient $D^{\mathrm{cc}}(z)=-1 / S_{0}^{\mathrm{cc}} M_{0}^{\mathrm{cc}}(z)$ can therefore be defined and a frequency dependent dispersion of the longitudinal modulus $K^{\mathrm{nn}}(z)-\lim _{q \rightarrow 0}\left[S_{q}^{\mathrm{nn}}-\left(S_{q}^{\mathrm{nc}}\right)^{2} / S_{q}^{\mathrm{cc}}\right]\left(1 / q^{2}\right) M_{q}^{\mathrm{nn}}(z)$, which is normalized 
by the bulk modulus of the liquid. Laplace transforms are defined as $f(z)=$ i $\int_{0}^{\infty} \mathrm{d} t \mathrm{e}^{\mathrm{i} z t} f(t)$; restoring the $\alpha$-time $\tau$ would lead to $f(z)=\tau \hat{f}(\hat{z})$.

For small wave vectors the generalized diffusion pole dominates the concentration fluctuations,

$$
F_{q}^{\mathrm{cc}}(z) \rightarrow \frac{-S_{0}^{\mathrm{cc}}}{z+q^{2} D^{\mathrm{cc}}(z)}\left[1+\mathcal{O}\left(q^{2}\right)\right], \quad \text { for } q \rightarrow 0 .
$$

Two poles appear in the total density fluctuations,

$$
\begin{aligned}
F_{q}^{\mathrm{nn}}(z) & \rightarrow \frac{-\left(S_{0}^{\mathrm{nc}}\right)^{2} / S_{0}^{\mathrm{cc}}}{z+q^{2} D^{\mathrm{cc}}(z)}\left[1+\mathscr{O}\left(q^{2}\right)\right]+\frac{-\left[S_{0}^{\mathrm{nn}}-\left(S_{0}^{\mathrm{nc}}\right)^{2} / S_{0}^{\mathrm{cc}}\right]}{z-1 / K^{\mathrm{nn}}(z)}\left[1+\mathcal{O}\left(q^{2}\right)\right], \\
\quad \text { for } q & \rightarrow 0
\end{aligned}
$$

The second pole is the analogue of the $\alpha$-peak in the coherent density fluctuations of a one component system; it is sometimes called Mountain pole [19]. The dispersion of the sound velocity follows from the $\alpha$-relaxation strength of the longitudinal modulus,

$$
K^{\mathrm{nn}}(t=0)=\frac{c_{\infty}^{2}-c_{0}^{2}}{c_{0}^{2}}=\frac{f_{0}^{\mathrm{nn}}-\left(S_{0}^{\mathrm{nc}}\right)^{2} / S_{0}^{\mathrm{cc}}}{S_{0}^{\mathrm{nn}}-f_{0}^{\mathrm{nn}}} .
$$

For small frequencies, $z \rightarrow 0+\mathrm{i} 0$, the diffusion $D^{\mathrm{cc}}(z) \rightarrow \mathrm{i} D^{\mathrm{cc}}$ and the longitudinal viscosity $K^{\mathrm{nn}}(z) \rightarrow \mathrm{i}\left(\eta_{1} / m n c_{0}^{2}\right)$ are obtained. The memory functions $M_{0}^{(\mathrm{s}) i}(z)$ determining the self-correlation functions reduce to the diffusion coefficients of species $i$ in this limit: $\mathrm{i} D^{(\mathrm{s}) i}=\lim _{z \rightarrow 0+\mathrm{i} 0} D^{(\mathrm{s}) i}(z)=$ $\lim _{z \rightarrow 0+\mathrm{i} 0} \lim _{q \rightarrow 0}\left[-1 / M_{q}^{(\mathrm{s}) i}(z)\right]$. For wave vectors $q$ close to $q_{\mathrm{p}}$, the position of the peak in the density structure factor $S_{q}^{\mathrm{nn}}, F_{q}^{\mathrm{nn}}(z)$ is dominated by the Mountain pole only.

\section{Numerical results}

For the specified mixture the critical parameter $\Gamma^{\mathrm{c}}$ and the exponent parameter $\lambda$ have been calculated before [12]. The present calculation gives almost identical results. The theoretical separation parameter $\sigma$ is found to be $\sigma \doteq C_{\Gamma}\left(\Gamma-\Gamma^{\mathrm{c}}\right) / \Gamma^{\mathrm{c}}$ with $\Gamma^{\mathrm{c}}=1.314$ and $C_{\Gamma} \approx 0.84$. The exponent parameter $\lambda=0.714$ leads to the exponent $\gamma=2.37$ and the von Schweidler exponent $b=0.62$. Further results of the MCT for the $\beta$-relaxation have been reported in $[12,13]$ and follow the scheme developed for simple liquids [1]. The results 
for the $\alpha$-relaxation will be presented comparing them to the known results for a HSS [6].

Fig. 1 shows the $\alpha$-relaxation strength $\int_{q}$ for density and concentration fluctuations. If both particle species were identical, then the total density fluctuations would correspond to the coherent density fluctuations of a one component system and the concentration fluctuations to the tagged particle dynamics. Cross correlations $F_{q}^{\mathrm{nc}}(t)$ would vanish. For the binary mixture
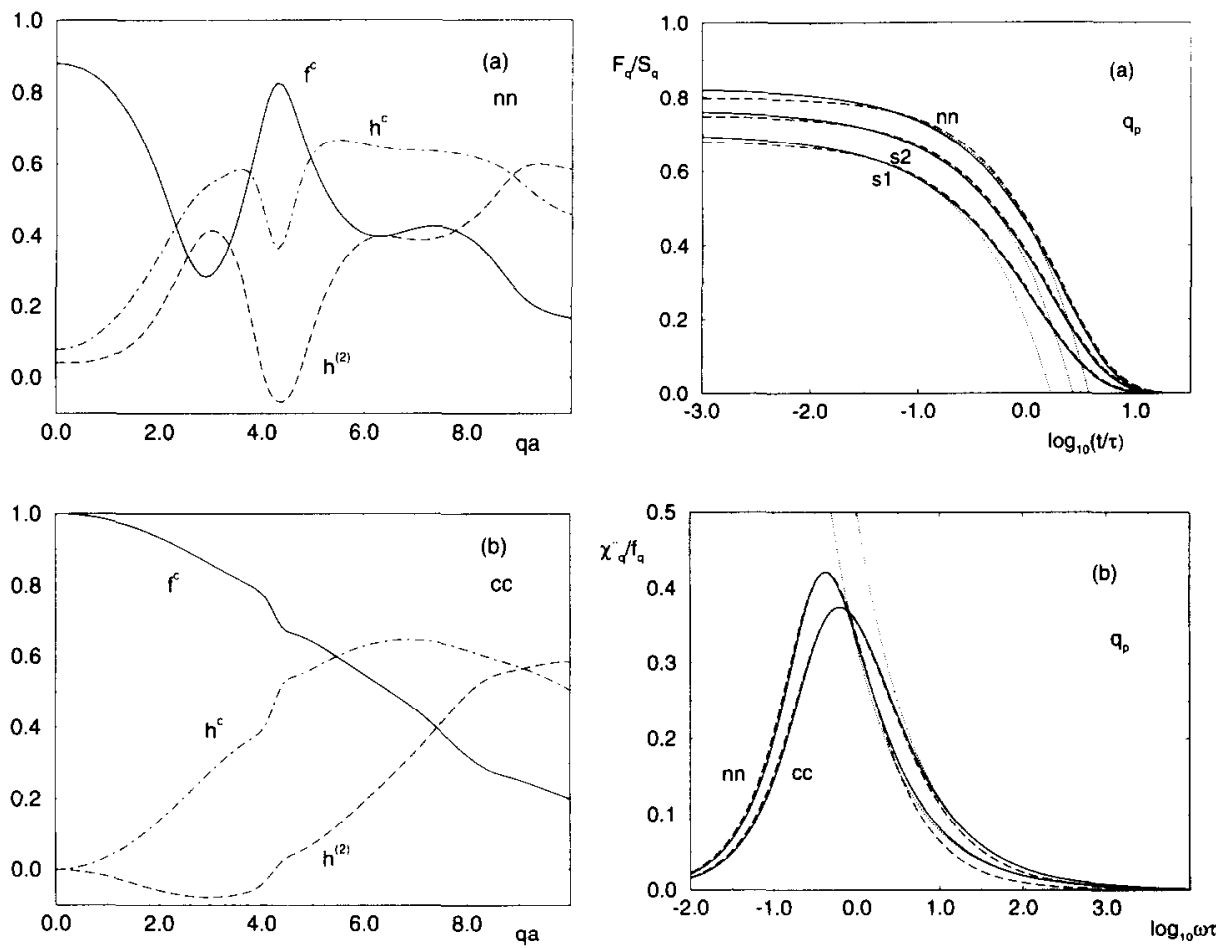

Fig. 1.

Fig. 2.

Fig. 1. Normalized $\alpha$-relaxation strength $f_{q} / S_{q}$ (solid line), critical amplitude $h_{q} / S_{q}$ (dash-dotted line) and coefficient $h_{q}^{(2)} / S_{q}$ (dashed line) of the $t^{2 b}$ term in (7) for density fluctuations in (a) and for concentration fluctuations in (b).

Fig. 2. (a) Normalized $\alpha$ - relaxation functions for density particle fluctuations, $F_{q_{p}}^{\mathrm{nn}}(t) / S_{q_{\mathrm{p}}}^{\mathrm{nn}}$, and single particle fluctuations $F_{q}^{(\mathrm{s}) i}(t), i=1,2$, at $q=q_{\mathrm{p}}=4.3 / a$ the peak in the density structure factor $S_{q}^{n n}$. The dotted lines are the von Schweidler asymptotes and the dashed lines are Kohlrausch functions fitted as explained in the text. The parameters are $\beta_{q_{p}}^{\mathrm{nn}}=0.84, \tau_{q_{\mathrm{p}}}^{\mathrm{nn}}=2.1, \beta_{q_{\mathrm{p}}}^{(\mathrm{s}) 1}=0.75$, $\tau_{q_{\mathrm{p}}}^{(\mathrm{s}) 1}=1.2, \beta_{q_{\mathrm{p}}}^{(\mathrm{s}) 2}=0.77$ and $\tau_{q_{\mathrm{p}}}^{(\mathrm{s}) 2}=1.7$. (b) Normalized density susceptibilities, $\chi_{q_{\mathrm{p}}}^{\mathrm{nn}}(\omega) / f_{q_{\mathrm{p}}}^{\mathrm{nn}}$, and concentration susceptibilities, $\chi_{q_{\mathrm{p}}}^{\mathrm{ccs}}(\omega) / f_{q_{\mathrm{p}}}^{\mathrm{cc}}$ at $q=q_{\mathrm{p}}$. Dotted lines represent the von Schweidler high frequency asymptote and broken lines the Kohlrausch fits. The parameters are identical to the ones used in (a) and $\beta_{q_{\mathrm{p}}}^{c c}=0.72$ and $\tau_{q_{\mathrm{p}}}^{c c}=1.3$. 
mainly $f_{q}^{\mathrm{nn}}$ is changed at small wave vectors $q$ due to the inter-diffusion pole appearing in $F_{q}^{\mathrm{nn}}(z)$. The dispersion of the longitudinal modulus and of the sound velocity, however, is only slightly smaller than in the HSS: $c_{\infty} \approx 1.20 c_{0}$ follows from (11) compared to $c_{\infty} \approx 1.54 c_{0}$ [6].

The corrections, i.e. $h_{q}^{i j(2)}$ in fig. 1 , in the expansion (7) to the von Schweidler asymptote are not small in general. Observing the von Schweidler law will therefore be difficult usually. For density fluctuations at the peak in the density structure factor $S_{q}^{\mathrm{nn}}, q=q_{\mathrm{p}} \approx 4.3 / a$, however, the asymptote follows the decay rather closely beyond its actual range of validity, see fig. 2. Fig. 2 presents typical $\alpha$-relaxation curves calculated in this binary system. They are non-exponential mainly due to the fractal short time expansion (7). The asymmetric shape can be well fitted by a Kohlrausch function (3) with parameters varying for different correlation functions. Especially the stretching exponents $\beta_{X}$, which are directly determined from the full width at half height of the susceptibilities $\chi^{\prime \prime}(\omega)$ as described in [6], vary with wave vector $q$. Systematic deviations of the Kohlrausch fits from the calculated curves at high frequencies or at short times result from the Kohlrausch exponents $\beta_{X}$ being larger than the von Schweidler exponent $b$ in general, $\beta_{X} \geqslant b$. These differences disappear for large $q$, where $b=\lim _{q \rightarrow \infty} \beta_{q}$ [20], and the MCT master curves become Kohlrausch functions as shown in fig. 3. The common low frequency asymptote, $\chi_{X}^{\prime \prime}(\omega) \propto \omega^{1}$ for $\omega \rightarrow 0$, of the calculated master curves is exemplified by a Debye fit to the susceptibilities of fig. 3 , which show the strongest stretching in this system.

Density-concentration cross correlation functions $F_{q}^{\mathrm{nc}}(t)$ exhibit peculiarities. The critical amplitudes $h_{q}^{\text {nc }}$ for these functions may become negative due to

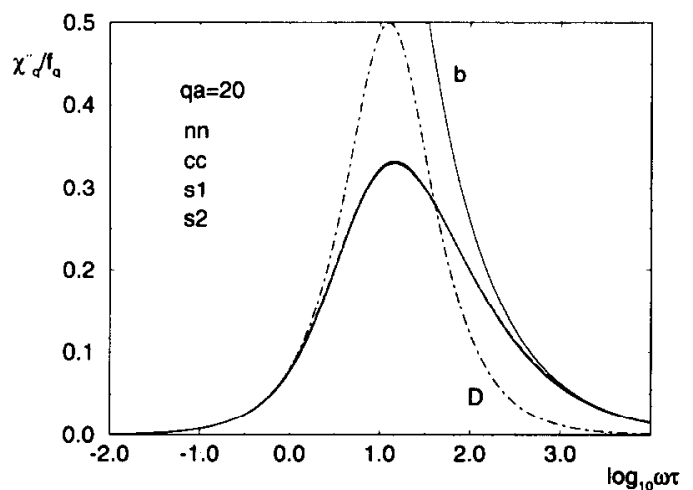

Fig. 3. Normalized $\alpha$-susceptibilities $\chi_{q}^{r^{\prime \prime}} / f_{q}^{\prime}$ for density-, concentration- and single particle dynamics, $r=\mathrm{nn}, \mathrm{cc}$, (s)1, (s)2, for a large wave vector, $q a=20$. The von Schweidler asymptotes are shown as dotted lines and Kohlrausch fits (coinciding with the theoretical curves) as dashed lines. The common fit parameters are $\beta_{q}^{\prime}=0.62$ and $\tau_{q}^{\prime}=0.05$. The chain curve corresponds to a Debye spectrum having the same low frequency asymptote as the calculated curves. 
negative vertices in (6b). For $q a=4.4$, for example, $F_{q}^{\mathrm{nc}}(t) / S_{q}^{\mathrm{nc}}$ does not decay monotonously. This demonstrates the importance of positive vertices $V_{q ; k p}$ in the proofs of the MCT for simple liquids [1].

For wave vectors $q$ smaller than $q_{\mathrm{p}}$ density fluctuations $F_{q}^{\mathrm{nn}}(t)$ show the expected two pole structure (10b). This is more obvious in the susceptibility spectra $\chi_{q}^{\mathrm{nn} \prime}(\omega)$, e.g. fig. $4 \mathrm{~b}$ for the wave vector $q=q_{p} / 2$ than in the corresponding correlators $F_{q}^{\mathrm{nn}}(t)$, see fig. 4a. The memory functions $\omega M_{q}^{\mathrm{nn} \prime \prime}(\omega)$
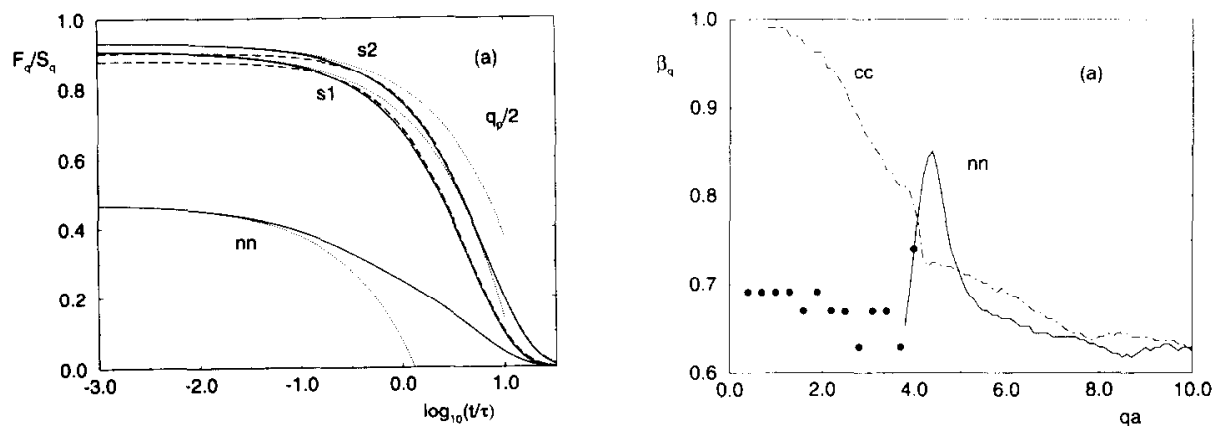

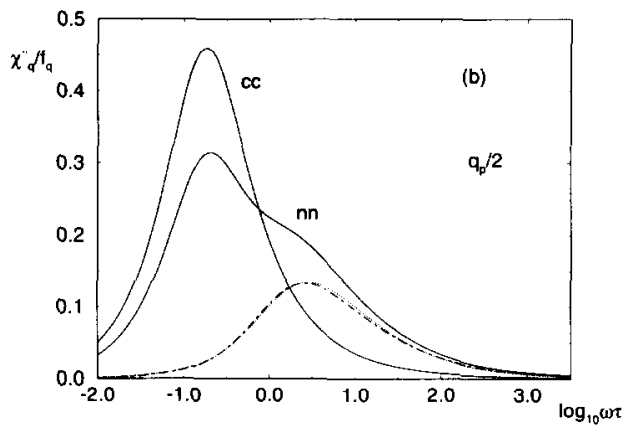

Fig. 4.

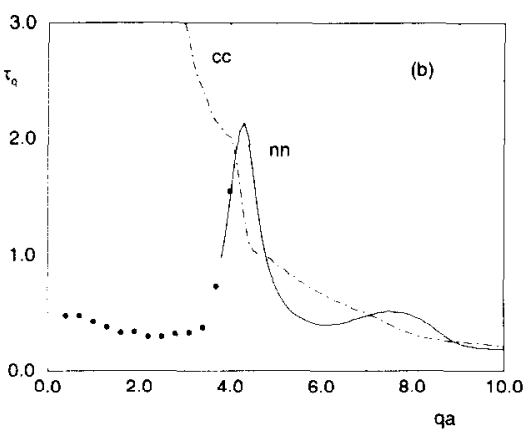

Fig. 5.

Fig. 4. (a) Normalized $\alpha$ - relaxation functions for density particle fluctuations, $F_{q}^{\mathrm{nn}}(t) / S_{q}^{\mathrm{nn}}$, and single particle fluctuations $F_{q}^{(\mathrm{s}) i}(t), i=1,2$, at $q=q_{\mathrm{p}} / 2$. The dotted lines are the von Schweidler asymptotes and the dashed lines are Kohlrausch functions fitted as explained in the text. The parameters are $\beta_{q_{\mathrm{p}} / 2}^{(\mathrm{s}) 1}=0.95, \tau_{q_{\mathrm{p}} / 2}^{(\mathrm{s}) 1}=4.8, \beta_{q_{\mathrm{p}} / 2}^{(\mathrm{s}) 2}=0.96$ and $\tau_{q_{\mathrm{p}} / 2}^{(\mathrm{s} / 2}=7.1$. (b) The solid lines show the normalized density susceptibilities, $\chi_{q_{\mathrm{p}} / 2}^{\mathrm{nn}^{\prime \prime}}(\omega) / f_{q_{\mathrm{p}} / 2}^{\mathrm{nn}}$, and concentration susceptibilities, $\chi_{q_{\mathrm{p}} / 2}^{c c^{\prime \prime}}(\omega) / f_{q_{\mathrm{p}} / 2}^{\mathrm{cc}}$ for $q=q_{\mathrm{p}} / 2$. The chain curve is the Mountain pole $\chi_{\mathrm{M}}$ contribution to $\chi^{\mathrm{nn}}: \chi_{q}^{\mathrm{M}}=$ $\chi_{q}^{\mathrm{nn}}(\omega) / f_{q}^{\mathrm{nn}}-0.63 \chi_{q}^{\mathrm{cc}}(\omega) / f_{q}^{\mathrm{cc}}$. It is fitted with a dotted Kohlrausch function with parameters $\beta_{q_{\mathrm{p}} / 2}^{\mathrm{M}}=0.67$ and $\tau_{q_{\mathrm{p}} / 2}^{\mathrm{M}}=0.30$.

Fig. 5. (a) Kohlrausch stretching exponents $\beta_{q}$ for the density (full line and circles) and the concentration fluctuations (dash-dotted curve). The fitting procedure and the meaning of the symbols are described in the text. (b) Relaxation times $\tau_{q}$ obtained by the Kohlrausch fits to the density susceptibilities (full line and circles) and concentration susceptibilities (dash-dotted) as specified in the text. 
and $\omega M_{q}^{\text {ccl' }}(\omega)$ show only a simple $\alpha$-relaxation peak very well described by Kohlrausch functions with e.g. $\beta^{M^{\mathrm{nn}}}=0.63$ and $\beta^{M^{\mathrm{cc}}}=0.62$ at $q_{\mathrm{p}} / 2$ [20]. The Mountain pole contribution $\chi_{q}^{\mathrm{M}}$, i.e. the second pole in (10b), can approximately be separated in $\chi^{\mathrm{nn}}$ by subtracting the inter-diffusion contribution; $\chi_{q}^{\mathrm{M}}=\chi_{q}^{\mathrm{nn} \prime \prime}(\omega) / f_{q}^{\mathrm{nn}}-\xi_{q} \chi_{q}^{\mathrm{ccl}}(\omega) / f_{q}^{\mathrm{cc}}$, with $\xi_{q} \approx 0.63$ at $q_{\mathrm{p}} / 2$. The relative amplitude $\xi_{q}$ is determined by optimizing the Debye low frequency asymptote. Eq. (10b) holds quantitatively for smaller wave vectors; at $q a=0.7$ it gives the relative amplitude $\xi_{q}=0.925$ within $2 \%$ error. At this small wave vector the Mountain- and the inter-diffusion peak are separated by ca. 2 decades in frequency [20].

In order to analyze the stretching of the density fluctuations for wave vectors $q$ less than $q_{\mathrm{p}}$ and to compare them with the results obtained in a HSS, the Mountain pole contributions $\chi_{q}^{\mathrm{M}}$ are studied for $q<q_{\mathrm{p}}$. The fitting procedure from [6] is used connecting $\beta$ with the full width at half height of the susceptibility spectrum. Fig. 5 collects the Kohlrausch parameters of the calculated and shown fits. Full circles represent results corresponding to $\chi^{\mathrm{M}}$. The scatter of the stretching exponents $\beta_{q}^{\mathrm{M}}$ at intermediate wave vectors, $1.5 \leqslant \mathrm{ya} \leqslant 4$ gives an error estimate. The parameters obtained from $\chi^{\mathrm{M}}$ match consistently to the ones obtained from the full density susceptibilities (shown as solid line in fig. 5) at wave vectors somewhat below $q_{\mathrm{p}}$. The results show qualitatively similar trends as in the HSS [6]. The close connection of $\beta_{q}$ and $\tau_{q}$ to the $\alpha$-relaxation amplitude $f_{q}$ and thereby to the structure factor $S_{q}$ can be noticed. Rather strong variations around the structure peak $q_{\mathrm{p}}$ arc found.

The stretching of the self correlation functions $F_{q}^{(\mathrm{s}) i}(t), i=1,2$, can be analyzed in the same way as in $F_{q}^{c c}(t)$. The Kohlrausch parameters are summarized in fig. 6 and corresponding fits are shown in figs. 2a, 3 and 4a. The
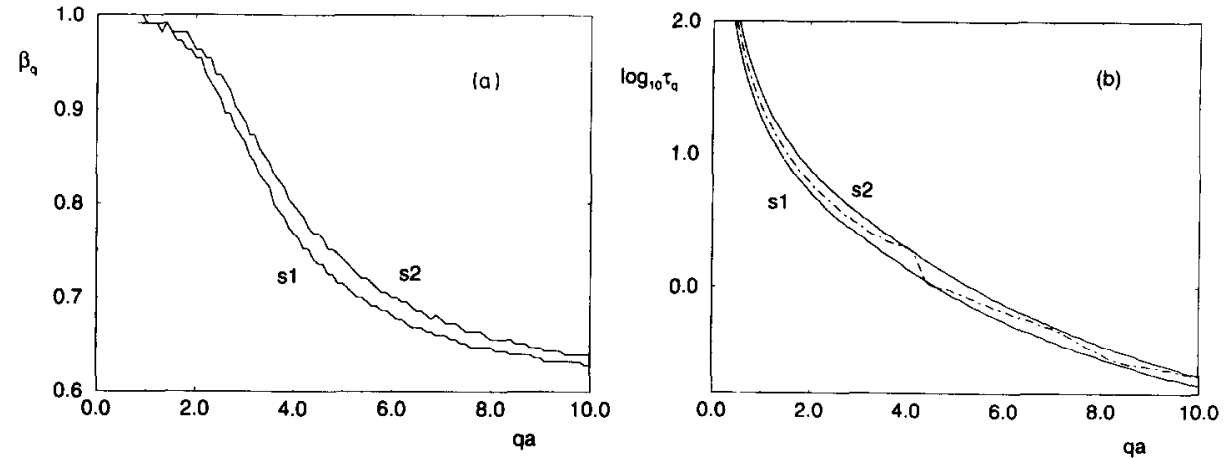

Fig. 6. Kohlrausch stretching exponents $\beta_{a}^{(s) i}$ in (a) and logarithm of the corresponding relaxation times $\tau_{a}^{(\mathrm{s}) i}$ in (b) for the tagged particle dynamics of species $i=1,2$; the same fitting procedure as in the collective dynamics is used. The relaxation time for interdiffusion fluctuations from fig. $5 \mathrm{~b}$ is included as dash-dotted curve in (b). 
smaller particles are somewhat faster and show stronger stretching. The relaxation times $\tau_{q}^{(\mathrm{s}) i}$ vary strongly with wave vector $q$; for small $q$ this variation is in accordance with a hydrodynamic behavior $q^{2} \tau_{q}^{(\mathrm{s}) i}=1 / D^{(\mathrm{s}) i}$. The diffusion coefficients can also be read off from the small frequency limits of the diffusion functions $D^{\mathrm{cc}}(\omega)$ and $D^{(\mathrm{s}) i}(\omega)$ shown in fig. 7,

$$
D^{\mathrm{cc}} \approx 0.044, \quad D^{(s) 1} \approx 0.055, \quad D^{(\mathrm{s}) 2} \approx 0.035 .
$$

The tagged particle motion can be well characterized by the van Hove self-correlation functions $F_{r}^{(\mathrm{s}) i}(t)$, the spatial Fourier (back) transforms of $F_{q}^{(s) i}(t) . P_{r}^{(s) i}(t)=4 \pi r^{2} F_{r}^{(\mathrm{s}) i}(t)$ is the probability of finding the particle of species $i$ after time $t$ in a distance $r$ from its starting point $r=0$ at $t=0$ [14]. If the motion was ruled by the hydrodynamic diffusion equation, a Gaussian result for $P_{r}(t)$ would be obtained [14],

$$
P_{r}^{\mathrm{H}}(t)=\frac{4 \pi r^{2}}{(4 D t)^{3 / 2}} \mathrm{e}^{-r^{2 / 4 D t}}, \quad \text { for hydrodynamic diffusion } .
$$

Due to the memory effects in the $\alpha$-master equations the $P_{r}^{(s) i}(t)$ deviate from this Gaussian result. For times small compared to the $\alpha$-relaxation time $\tau$ the particles are localized during the $\beta$-process. The root mean square displacements follow from the $\alpha$-amplitudes of the incoherent moduli $M_{0}^{(\mathrm{s}) i}(t=0)$,

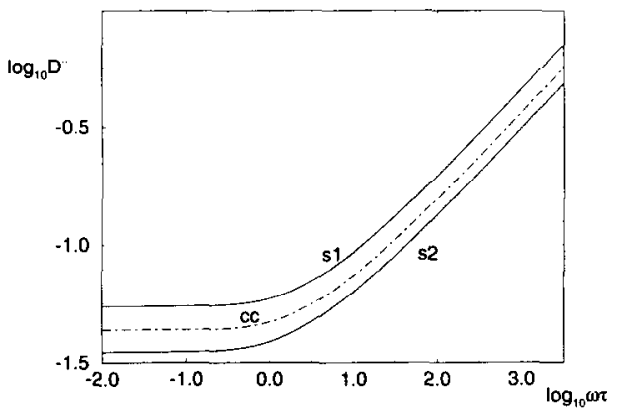

Fig. 7.

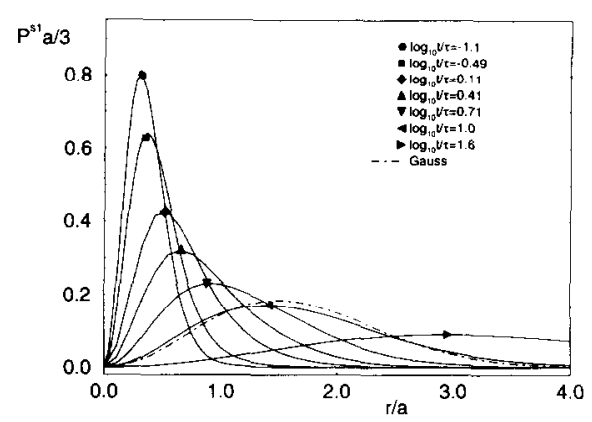

Fig. 8 .

Fig. 7. Logarithm of the frequency dependent inter diffusion coefficients, $D^{\mathrm{cc}}(\omega)$, and tagged particle diffusion coefficients, $D^{(s) i}(\omega)$.

Fig. 8. (Scaled) probability density $\frac{1}{3} a P_{r}^{(s) 1}(t)$ for particle species 1 and times $\log _{10}(t / \tau)=-1.1$, $-0.49,0.1,0.4,0.7,1.0$ and 1.6. The dash-dotted curve is the Gaussian diffusion (13) with the correct diffusion coefficient from (12) and evaluated at the corresponding time $\log _{10}(t / \tau)=1.0$. 


$$
\sqrt{\left\langle\left(\Delta r_{1}^{(\mathrm{s})}\right)^{2}\right\rangle} \approx 0.14 \mathrm{a}, \quad \sqrt{\left\langle\left(\Delta r_{2}^{(\mathrm{ss}}\right)^{2}\right\rangle} \approx 0.12 a .
$$

These values lie close to the result in a HSS [6]. The $\alpha$-relaxation strength $f_{r}^{(\mathrm{s}) 2}$ exhibits a qualitative deficiency of the MCT in this soft sphere mixture. For $r / a \approx 0.7, f_{r}^{(\mathrm{s}) 2}$ becomes negative; the error is quantitatively less than $2 \%$ of the maximal value of $f_{r}^{(\mathrm{s}) 2}$. For times long compared to the $\alpha$-relaxation time the diffusion result (13) with the diffusion coefficient from (12) describes the results for $P_{r}^{(s) i}(t)$ rather well; see fig. 8.

\section{Comparison with $\mathrm{MD}$ results}

The MCT calculations give a rather detailed picture of the dynamics of the supercooled binary liquid below the critical coupling $\Gamma^{\mathrm{c}}$ which can be compared with the existing MD data.

The calculated $\beta$-relaxation has - as mentioned above - been compared to the total density fluctuations at the structure peak $q_{\mathrm{p}}$ [13]. The large fit intervals using the von Schweidler asymptote are possible there due to the fortunate cancellation effects shown in fig. 2. The published MD results for density and single particle correlators at two wave vectors, $q=q_{\mathrm{p}}$ and $q=q_{\mathrm{p}}$ ' 2 , show qualitative agreement with the calculated curves $[9,10]$. The MD curves show stretching, parametrized by $\beta_{q_{\mathrm{p}}}^{(s) 1}=0.62, \beta_{q_{\mathrm{p}}}^{\mathrm{nn}}=0.81 \pm 0.08$ and $\beta_{q_{\mathrm{p}} / 2}^{(\mathrm{s}) 1} \approx 1.0$ [10], which is compatible with our results, if the fitting uncertainties and the problems of separating $\alpha$ - and $\beta$-dynamics are taken into account. If more MD runs closer to the transition point were available, $\alpha$-master scaling of curves for different parameters $\Gamma$ would allow more stringent comparisons.

The calculated bulk modulus at high frequencies, $\omega \tau \gg 1$, is close to the one found in the HSS [6] and slightly overestimates the MD results $K^{\mathrm{nn}}(t=0) \approx 0.3$ [11]. The stated qualitative failure of the MCT to approximate this quantity is due to an erroneous neglect of the inter-diffusion contributions to $f_{0}^{\mathrm{nn}}$ [11].

For times short compared to the $\alpha$-relaxation time $\tau$ the calculated tagged particle dynamics renders mean square displacements which lie close to the reported MD values $\sqrt{\left(\Delta r^{(s) i}\right)^{2}} \approx 0.11 a$ [9]. For times long compared to $\tau$ the MD simulations also find the tagged particle motion to obey a simple diffusion law, the ratio of the diffusion coefficients $D^{(\mathrm{s}) 2} / D^{(\mathrm{s}) 1}=0.68 \pm 0.08[10]$ corresponds to the theoretical value $D^{(\mathrm{s}) 2} / D^{(\mathrm{s}) 1}=0.64$.

The crossover in the tagged particle motion $[9,10]$ for coupling parameters close to $\Gamma^{\mathrm{c}}$ has been discussed in terms of the localized $\beta$-relaxation of the MCT in [13]. The present calculation does not change this discussion as it obtains similar non ergodicity parameters $f_{q}^{(\mathrm{s}) i}$ and critical amplitudes $h_{q}^{(\mathrm{s}) i}$ as used in [13]. 


\section{Conclusions}

The MCT results for the $\alpha$-relaxation in an undercooled binary soft-sphere mixture exhibit analogous properties as found in one component systems. The $\alpha$-relaxation is a non-exponential decay process which shows non-universal features. It can be described by an asymptotic scaling law, which connects the $\alpha$-dynamics with the equilibrium structure of the system. The $\alpha$-master curves for all dynamic quantities share a common von Schweidler short time power law with exponent $b$ depending on the static partial structure factors $S^{i j}(q)$ at $T_{\mathrm{c}}$. The master functions for different variables show different stretching which depends sensitively on the matrix $S_{q}$. The overall shape is in general well represented by Kohlrausch functions with varying exponents $\beta_{X}$ and relaxation times $\tau_{X}$. Systematic deviations at high frequencies due to $\beta_{X}>b$ are observed. The presented results for the simple binary mixture allow quantitative tests of the underlying microscopic MCT. There are some specific differences to one component systems. Mainly for small wave vectors a double peak structure in the density-density spectrum appears due to the interference of the hydrodynamic interdiffusion and the Mountain peak.

\section{Acknowledgements}

We cordially thank W. Götze for useful discussions and encouragement. We thank J.L. Barrat for providing the program for calculating $S_{q}$. One of us (A.L.) acknowledges financial support from the Deutsche Forschungsgemeinschaft.

\section{References}

[1] W. Götze, in: Liquids, Freezing and Glass Transition, J.P. Hansen, D. Levesque and J. Zinn-Justin, eds. (North-Holland, Amsterdam, 1991) p. 287.

[2] W. Götze and L. Sjögren, Rep. Prog. Phys. 55 (1992) 241.

[3] W. Götze and L. Sjörgen, Z. Phys. B 65 (1987) 415.

[4] L. Sjögren, Z. Phys. B 65 (1987) 415.

[5] M. Fuchs, W. Götze, I. Hofacker and A. Latz, J. Phys. Cond. Matt. 3 (1991) 5047.

[6] M. Fuchs, I. Hofacker and A. Latz, Phys. Rev. A 45 (1992) 898.

[7] W. van Megen, S.M. Underwood and P.N. Pusey, Phys. Rev. Lett. 67 (1991) 1586; W. van Megen and S.M. Underwood, Phys. Rev. E 47 (1993) 248; Phys. Rev. Lett. 70 (1993) 2766.

[8] B. Bernu, J.P. Hansen, Y. Hiwatari and G. Pastore, Phys. Rev. A 36 (1987) 4891.

[9] J.N. Roux, J.L. Barrat and J.P. Hansen, J. Phys. Cond. Matt. 1 (1989) 7171.

[10] J.L. Barrat, J.N. Roux and J.P. Hansen, Chem. Phys. 149 (1990) 197. 
[11] J.N. Roux, in: Slow Dynamics in Condensed Matter, K. Kawasaki, T. Kawakatsu and M. Tokuyama, eds. (AIP, New York, 1992) p. 173.

[12] J.L. Barrat and A. Latz, J. Phys. Cond. Matt. 2 (1990) 4289.

[13] M. Fuchs, W. Götze, S. Hildebrand and A. Latz, Z. Phys. B 87 (1992) 43.

[14] J.P. Hansen and I.R. McDonald, Theory of Simple Liquids, 2nd ed. (Academic Press, London, 1986).

[15] F.J. Rogers and D.A. Young, Phys. Rev. A 30 (1984) 999.

[16] J. Bosse and T. Munakata, Phys. Rev. A 25 (1982) 2763.

[17] L. Sjögren and F. Yoshida, J. Chem. Phys. 77 (1982) 3703.

[18] J. Bosse and J.S. Thakur, Phys. Rev. Lett. 59 (1987) 998.

[19] R.D. Mountain, J. Res. Natl. Bur. Stand. A 70 (1966) 207.

[20] M. Fuchs, PhD. Thesis, TU-Munich (1993). 